\title{
ESTIMATIVA DA ÁREA FOLIAR DE PLANTAS DANINHAS: POAIA-BRANCA ${ }^{1}$
}

\author{
RICARDO R. ROSSETO ${ }^{2}$, ROBINSON L.C. M. PITELLI², ROBINSON A. PITELLI ${ }^{3}$
}

\section{RESUMO}

A poaia-branca (Richardia brasiliensis Gomez) é uma das principais plantas que infestam espontaneamente os agroecossistemas na América do Sul e, com freqüência, atingem elevadas densidades populacionais, provocando sérios prejuízos aos agricultores. Nos estudos envolvendo a biologia e o controle de plantas daninhas, a área foliar é uma das mais importantes características a sere $\mathrm{m}$ avaliadas, mas tem sido pouco estudada porque sua determinação exige equipamentos sofisticados ou utiliza técnica

destrutiva. Visando obter equações que permitissem a estimativa da área foliar desta planta daninha utilizando características lineares do limbo foliar, facilmente mensuráveis em plantas no campo, foram estudadas correlações entre a área foliar real e as seguintes característic as das folhas: comprimento ao longo da nervura principal $(\mathrm{C})$, largura máxima do limbo (L) e o produto CxL. Para tanto, foram mensurados os limbos de 500 folhas coletadas em diversas épocas, locais e culturas, em plantas que apresentavam bom aspecto sanitário e nutricional. Todas as equações, lineares simples, geométricas e exponenciais, permitiram boa estimativa da área foliar $(A F) d a$ poaia-branca. Do ponto de vista prático, sugere-se optar pela equação linear simples envolvendo o produto CxL, a qual apresentou o menor QM Resíduo. As sim, a estimativa da área foliar de $R$. brasiliensis pode ser efetuada pela equação $A F=0.5899(C x L)$, com coeficiente de determinação $\left(\mathrm{R}^{2}\right)$ de valor 0.9886 .

Palavras chave: Richardia brasiliensis, planta daninha, área foliar.

\section{ABSTRACT}

\section{Leaf area determination of weeds: Brazil callatity.}

Brazilian pusley (Richardia brasiliensis) is one of the most important weeds infesting orchards, crops and pastures in South America. Frequently, their populations are dense enough to promote significant losses to the farmers. In the studies on the biology and control of the weeds, the leaf area determination is fundamental, but commonly requires sophisticated and expensive equipment or destructive techniques. So, the present research was set up aiming to obtain a suitable equation for $R$. brasiliensis leaf area estimation from the linear leaf measures, such as the leaf length in the mid rib direction $(\mathrm{C})$, the maximum leaf width (L), and the relation $C x L$. The measures were done in 500 leaves collected from plants infesting diffe rent places and crops and showing suitable nutritional and health conditions. All the equations, linear, geometric, and expon ential, permitted suitable estimates of the $R$. brasiliensis leaf area $(A F)$. The suggested equations was $A F=0.5899(C x L)$, because it is easy to use, showed the smallest Stand Error, and the determination coefficient was high, $\mathrm{R} 2=0.9886$. leaf area.

\footnotetext{
1 Recebido para publicação em 10/01/97 e na forma revisada em 23/05/97.

2 Acadêmico em Agronomia, FCAV/UNESP, 14870-000, Jaboticabal, SP, Brasil.
}

3 Professor Titular, FCAV/UNESP, 14870-000, Jaboticabal, SP, Brasil. 


\section{INTRODUÇÃO}

A poaia-branca (Richardia brasiliensis Gomez) é uma das mais importantes entre as plantas que infestam espontaneamente as áreas agrícolas do Brasil. É uma planta anual nativa da América do Sul, ocorrendo desde a Cordilheir a dos Andes até a Costa Atlântica. No Brasil, tem va sta di stribui ção ge og ráfic a, com maior ocorrência em regiões agrícolas do Centro-Oeste, Sudeste e Sul. É mais comum em solos medianos a leves, com boa umidade mas não encharcados; seu desenvolvimento é estimulado com boa iluminação, sendo mais agressiva em solos abertos. Esta planta daninha infesta pastagens, pomares e lavouras, nas quais promove intensa interferência competitiva, especialmente no início do ciclo de culturas de verão (Kissmann \& Groth, 1994). Esta espécie tem sido selecionada por herbicidas e pela adoção do sistema de plantio direto, na região sul do país (Pitelli, 1991). Na Argentina, a poaia-branca é uma planta daninha comum, especialmente em pomares, ocorrendo desde o noroeste da província de Entre-Rios até Corrientes, onde infesta principalmente plantações de tabaco (Marzocca et al., 1979). Além dos países da América do Sul, tem sido citada como planta daninha na Rodésia, Suazilândia, Havaí, Indonésia, Africa do Sul, EUA e Quênia (Holm et al., 1991).

Considerando a importância desta planta daninha, há grande necessidade de estudos básicos envolvendo aspectos relacionados à reprodução, cres ciment o, de se nv olvi mento, ex igênci as nutricionais, respostas aos sistemas de controle e outros. Na maioria destes estudos, o conhecimento da área foliar é fundamental. É uma das características mais difíceis de serem mensuradas, porque normalme nte requer equipamentos caros ou utiliza técnica destrutiva, como comentam Bianco et al. (1995). Uma das opções interessantes constitui a utilização de equações que permitam a estimativa da área foliar a partir de medidas lineares da folha. Este método já foi utilizado com sucesso para inúmeras plantas cultivadas e plantas daninhas, como Wissadula subpeltata (Kuntze) Fries (Bianco et al., 1983b), Senna obtusifolia Irwin \& Barneby (Peressin et al., 1984), Amaranthus retroflexus L. (Bianco et al., 1995), plantas que não apresentam heterofilia. Este mé todo tem a vantagem de permitir a estimativa da área foliar de uma mesma planta em diferentes épocas. O presente trabalho teve o objetivo de determinar uma equação adequada para a estimativa da área foliar de $R$. brasiliensis por intermédio de medidas lineares do limbo foliar.

\section{MATERIAL E MÉTODOS}

Foram coletadas 500 folhas de $R$. brasiliensis em plantas sujeitas às mais diversas condições que a espécie é susceptível de ocorrer em lavouras e pomares, sendo colhidas apenas de plantas que apresentavam bom aspecto sanitário e nutricional. Foram consideradas todas as folhas da planta, de scartando-se apen as aquelas que apresentavam deformações decorrentes da ação de agentes externos, como pragas e moléstias.

$\mathrm{Na}$ fase de coleta dos dados, foram realizadas rápidas excursões ao campo, coletandose de 20 a 50 folhas, as quais eram levadas ao laboratório para obtenção dos valores necessários para a pesquisa. Este procedimento procu rou evitar que a perda de turgidez das folhas pudesse alterar os resultados. Cada excursão foi realizada em épocas e locais diferentes.

No laboratório, em cada folha foram determinados: o comprimento ao longo da nervura principal $(\mathrm{C})$, a largura máxima perpendicular à nervura principal $(L)$ e a área foliar real $(\mathrm{Sr})$. Estas determinações foram efetuadas com auxílio do aparelho "Area Meter Li-Cor 2000".

A seguir, foram efetuados estudos de reg ress ão entre a área fol iar real $(S r)$ e o comprimento (C), a largura da folha $(L)$ e relação $C x L$. Foram estudadas equações lineares $\mathrm{Y}=\mathrm{A}+$ $\mathrm{BX}, \mathrm{Y}=\mathrm{BX}$, geométricas $\mathrm{Y}=\mathrm{AX}^{\mathrm{B}}$ e exponencial $\mathrm{Y}=\mathrm{AB}^{\mathrm{X}}$. O valor $\mathrm{Y}$ estima a área da folha em função de $X$, cujos valores podem ser o comprimento (C), a largura ( $L$ ) ou o produto $C x L$.

\section{RESULTADOS E DISCUSSÃO}

As plantas de poaia-branca apresentam folhas simples e opostas. Há um par de folhas em cada nó do caule, observando-se a presença de estípulas interpeciolares. O pecíolo é curto. $\mathrm{O}$ 
limbo foliar apresenta forma ovada a lanceolada, oblonga, elíptica ou debilmente lanceoladainvertida e coloração verde-escura. O caráter lan ceolado é atenuado na base do limbo. Kissmann \& Groth (1994) ainda acrescentam aspectos relacionados às nervuras, que são mais proeminentes na face dorsal. Ocorre tênue pilosidade na face ventral e sobre as nervuras na face dorsal. Não foi observada qualquer variação de forma nas folhas que pudesse ser caracterizada como heterofilia.

$\mathrm{Na}$ Tabela 1 es tão ap res entados os resultados referentes aos estudos de regressão efetuados com as comparações da área foliar real (Sr) e as medidas lineares de comprimento (C), largu ra $(L)$ e o produto do comprime nto pela la rgura da folh a $(C x L)$. Todas as equações apresentadas na tabela permitiram estimativas satisfatórias da área foliar de $R$. brasiliensis, com coeficientes de correlação acima de 0,94.

TAB ELA 1. Equações de re gre ssão para estimativa da área foliar de poaia-branca (Richardia brasiliensis) utilizando medidas lineares do limbo.

\begin{tabular}{llcccc}
\hline \multicolumn{1}{c}{ Medida } & \multicolumn{1}{c}{ Equação } & S.Q. Resíduo & G.L. Resíduo & $\mathbf{r}^{2}$ & Q.M. Resíduo \\
\hline $\mathbf{C}$ & $\mathbf{A F}=0.8186+1.1342 \mathbf{C}$ & 111.9385 & 498 & 0.9436 & 0.2248 \\
$\mathbf{L}$ & $\mathbf{A F}=0.7713+1.3955 \mathbf{L}$ & 104.6097 & 498 & 0.9474 & 0.2106 \\
$\mathbf{C} \times \mathbf{L}$ & $\mathbf{A F}=0.1002+0.5721 \mathbf{C x L}$ & 23.2097 & 498 & 0.9886 & 0.0466 \\
$\mathbf{C} \times \mathbf{L}$ & $\mathbf{A F}=0.589988(\mathbf{C x L})$ & 24.5459 & 499 & 0.9886 & 0.0492 \\
$\mathbf{C}(\mathrm{geom})$ & $\mathbf{A F}=0.3135 \times \mathbf{C}^{1.1714}$ & 97.5443 & 498 & 0.9507 & 0.1958 \\
$\mathbf{L}$ (geom) & $\mathbf{A F}=1.1604 \times \mathbf{L}^{1.1898}$ & 81.4288 & 498 & 0.9593 & 0.1635 \\
$\mathbf{C}$ (expon) & $\mathbf{A F}=0.3978 \times 1.1686^{\mathbf{C}}$ & 208.3554 & 498 & 0.9593 & 0.4184 \\
$\mathbf{L}$ (expon) & $\mathbf{A F}=0.3258 \times 1.4727^{\mathbf{L}}$ & 125.5514 & 498 & 0.9463 & 0.2521 \\
\hline
\end{tabular}

O comprimento das folhas ao longo da nervura principal variou entre 1,2 e $6,7 \mathrm{~cm}$, com valor médio de $3,17 \mathrm{~cm}$. A largura máxima das folh as variou entre 0,50 e $2,3 \mathrm{~cm}$, com val or médio de $1,2 \mathrm{~cm}$. Para a área foliar real, os valores vari aram entre 0,40 e $8,47 \mathrm{~cm}^{2}$ e média de 2,45 $\mathrm{cm}^{2}$. Numa classificação em diferentes faixas de tamanho das folhas, observou-se que $9.4 \%$ apresentaram área foliar entre $\mathrm{O}$ e $1 \mathrm{~cm}^{2}, 39.0 \%$ entre 1 e $2 \mathrm{~cm}^{2}, 24.8 \%$ entre 2 e $3 \mathrm{~cm}^{2}, 11.6 \%$ entre 3 e $4 \mathrm{~cm}^{2}, 7.8 \%$ entre 4 e $5 \mathrm{~cm}^{2}, 5.0 \%$ entre $5 \mathrm{e} 6 \mathrm{~cm}^{2}, 1,8 \%$ entre 6 e $7 \mathrm{~cm}^{2}, 0.2 \%$ entre 7 e 8 $\mathrm{cm}^{2}$ e $0.4 \%$ entre 8 e $9 \mathrm{~cm}^{2}$. Houve uma predominância de folhas de área na faixa entre $\mathrm{O}$ e $5 \mathrm{~cm} 2$

Os maiores valores do coeficiente de co rrelação e os menores valores do quadrado médio do resíduo foram observados para as regressões lineares simples entre a área foliar real e o produto do comprimento pela largura da folha, indicando serem as equações que permitem estimativas mais acuradas da área foliar da planta daninha. A equação linear simples com a reta passando pela origem é a mais recomendada, pois mantém o valor do coeficiente de correlação, não al te ra expressivamente o quadrado médio do resíduo e é de mais fácil utilização. Assim, a estimativa da área foliar da poaia-branca pode ser feita pela equação $A F=0.589988 \times(C x L)$, ou seja, $58,99 \%$ do produto do comprimento pela la rg ura máxima da folha. O coeficiente de determinação de valor 0,9886 indica que 98,86\% dos valores medidos, puderam ser explicados pela equação acima.

Na Figura 1 estão graficamente representados os valores obtidos para o produto do comprimento pela largura do limbo e o correspondente valor da superficie foliar e, ta mbém, a repres en tação gráfic a da equação indicada para a estimativa da área de folhas de $R$. brasiliensis. Pode-se observar a pequena dispersão dos dados em relação à reta obtida. $\mathrm{O}$ valor ora obtido é equivalente ao encontrado para Amaranthus retroflexus (Bianco et al., 1995) e 
inferiores aos observados para cultivares de laranjeira (Citrus sinensis Osbeck) (Bianco et al., 1983a) e folíolos de Senna obtusifolia (Peressin et $a l ., 1984)$. Tanto as folhas de $A$. retroflexus como de $R$. brasiliensis apresentam formas mais alongadas, explicando as diferenças observadas para as outras duas espécies.

Os resultad os alca nçad os pe rmitem concluir que é possível a estimativa da área foliar de Richardia brasiliensis através da equação $\mathrm{AF}=$ $0.589988 \times(\mathrm{CxL})$, com coeficiente de determinação de $98.86 \%$.

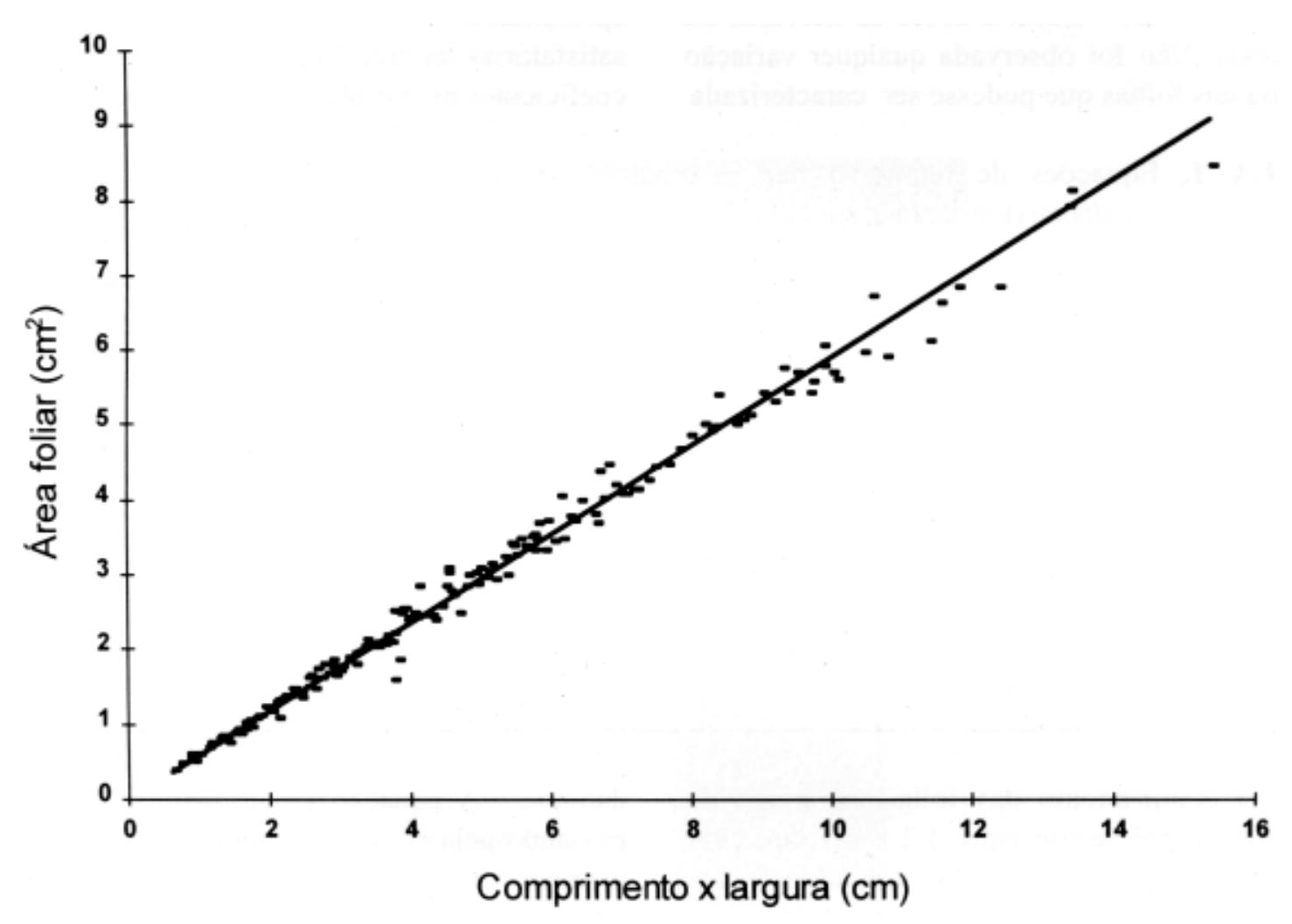

FIGURA 1. Representações gráficas dos produtos do comprimento pela largu ra do limbo e os correspondentes valores da área foliar de Richardia brasiliensis e da equação de regressão indicada para estimativa da área foliar da planta daninha.

\section{REFERÊNCIAS BIBLIOGRÁFICAS}

BIANCO, S., PIT ELLI R.A., PAVANI, M.C.M.D., SILVA, R.C. Estimativa da área foliar de quatro cultivares de laranjeiras (Citrus sinens is Osbeck.). Cien. Agron., v.2, n.1, p.129-134, 1983a.

BIANCO, S., PITELLI R.A., PAVANI, M.C.M.D., SILVA, R.C. Estimativa da área foliar de plantas daninhas. XIII.
Amaranthus retroflexus L. Ecossistema, v.20, p.5-9, 1995.

BI ANCO, S., PITELLI R.A., PERECIN, D. Método para estimativa da área foliar de plantas daninhas. 2. Wissadula subpeltata (Kuntze) Fries. Planta Daninha v.6, n.1, p.21-24, 1983b.

HOLM, L. G., PA NCHO, J. V., HERB ER GER, J.P., PLUCKNET, D.L. A Geographical 
Atlas of World Weeds. Malabar, Krieger Publishing Company, 1991. 391 p.

KISSMANN, K., GROTH, D. Plantas Infestantes e Nocivas. Tomo II. São Paulo, BASF Brasileira, 1994. 603 p.

MARZOCCA, A., MARSICO, O.J.V., PUERTO, O. Manual de Malezas. Buenos Aires, Editorial Hemisferio Sur, 1979. 564p.
PERESSIN, V.A., PITELLI, R.A., PERECIN, D. Método para estimativa da área foliar de plantas daninhas.4. Cassia tora L.. Planta Daninha, v.7, n.2, p.48-52, 1984.

PITELLI, R.A. We ed -so ybea n in te rferen ce studies in Brazil. In: COOPING, L.G., GREEN, M.B., REES, R.T. Pest Man agement in Soybea n. Lo nd on, Elsevier Publishers, Ltd., 1991. p.282-289. 